\title{
Surface tension determination using data of the evolution of thermocapillary deformations in a locally heated liquid layer
}

\author{
Ella Barakhovskaia ${ }^{1,2, *}$ and Igor Marchuk ${ }^{1,2}$ \\ ${ }^{1}$ Novosibirsk State University, Pirogova Str. 2, Novosiborsk, 630090, Russia \\ ${ }^{2}$ Kutateladze Institute of Thermophysics, Russian Academy of Sciences, Prosp. Lavrentyev 1, \\ Novosibirsk, 630090, Russia
}

\begin{abstract}
Identifying liquids in chemical technologies and in the oil industry is an actual problem. Such identifications can be made using individual characteristics of thermocapillary flows and deformations in the horizontal layers of the investigated liquids. Numerical algorithms and calculating code have been developed for solving inverse problems for identifying properties of liquid. As additional information for inverse problem a function of thermocapillary response is used. The algorithm for solving inverse problem is based on solving problem of minimizing the residual function. The minimum is obtained numerically by the Newton's method. Numerical calculations have been made for silicone oil. Thermocapillary responses have been calculated for different values of the coefficients. The developed algorithms have been tested on synthetic data, which have been obtained from the solution of direct problems. The developed algorithms have shown a good convergence and efficiency.
\end{abstract}

\section{Introduction}

Identifying liquids in chemical technologies and in the oil industry is a topical issue in the present days. Such identification can be made according to individual characteristics of thermocapillary flows and deformations in the horizontal layers of the investigated liquids. To a large extent the thermocapillary effect occurs while thin liquid layers are locally heated [1]. Thermocapillary effect can be successfully used to determine the properties and the thickness of the thin locally heated liquid layer [2].

The inverse problem of thermocapillary convection consists in determining the surface tension coefficients $\sigma$ and the temperature coefficient of the surface tension $\sigma_{\mathrm{T}}$ according to thermocapillary response that has been measured in the experiment. Thermocapillary response is the time dependence of the laser beam diameter reflected from the surface of the liquid and projected onto the screen, Fig. 1a.

A method for measuring the thickness of a liquid layer using a laser-induced thermocapillary effect was proposed in paper [2], and a method for identifying liquids was proposed in paper [3]. This paper presents a method for calculating $\sigma$ and $\sigma_{\mathrm{T}}$ coefficients

* Corresponding author: ella94@bk.ru 
based on the data about the dynamics of deformations in a thin locally heated horizontal liquid layer.

\section{Problem statement}

Solving the inverse problem of thermocapillary convection is based on results of studying the direct problem of thermocapillary deformation of thin horizontal film [5].

The model considers a thin horizontal liquid layer on the substrate with local heater. Liquid is viscous and incompressible. Heater is thin and uniform. Geometry and system conditions are axisymmetric. At the time $\mathrm{t}=0$ the heater is activated and starts producing the pulse heating power from the substrate side during 1 second. Shear stress occurs on the liquid surface caused by the heterogeneity of its temperature. This causes thermocapillary flow and deformations of the liquid surface.

Two-dimensional unsteady problem is considered. The direct and inverse problems have been solved numerically in axisymmetric statement. The thin layer approximation model is used [4]. Capillary pressure, viscosity and gravity are taken into account. Evaporating rate is neglected because of chosen type of liquid (silicone oil dimethylpolysiloxane, PMS-5). Initially the liquid layer has flat surface and uniform constant temperature. The initial thickness of the liquid layer varies from $100 \mu \mathrm{m}$ to a few millimeters. Schematic of the system is shown in Fig. 1a.

\section{Measuring the thermocapillary response}

The inverse problem of thermocapillary convection consists on determining the surface tension coefficients $\sigma$ and $\sigma_{\mathrm{T}}$. A function of thermocapillary response is used as additional information for solving inverse problem:

$$
d S p(t)=\max _{r \in\left[0, d_{l} / 2\right]}|2 \cdot| r-(d-h(t, r)) \cdot \operatorname{tg}\left(2 \operatorname{arctg}\left(h_{r}^{\prime}(t, r)\right)\right)||
$$

where $d l$ - is the diameter of a laser beam, $h(t, r)$ - is the film thickness, $h_{r}^{\prime}(t, r)$ - is the first derivative of thickness, $d$ - is the distance to the screen.

Thermocapillary response function is a special experimentally measured time dependent function of laser beam diameter, reflected from the liquid surface and projected on a screen, Fig. 1a.

Liquid flow and deformations of its surface and, as a result, thermocapillary response are determined by the liquid properties, as well as the properties of the cuvette material, heater and heating characteristics. For different liquids at other factors being equal, the kind of thermocapillary response is significantly different.

\section{Mathematical formulation}

Dynamics of thin films is well described by the evolution equation, which is obtained in the lubrication approximation theory $[5,6]$ :

$$
h_{t}+\operatorname{divq}=0
$$

where, $h_{t}-$ is the velocity of the surface, $\boldsymbol{q}=\frac{h^{3}}{3 \mu} \boldsymbol{f}+\frac{h^{2}}{2 \mu} \boldsymbol{\tau}-$ is the liquid flow rate along the $r$ direction, $\boldsymbol{f}=\operatorname{grad}(\rho \boldsymbol{g} h+\sigma H)$ - is the gradient of the modified pressure, $\boldsymbol{\tau}=\sigma_{T} \operatorname{grad} T$ - is the thermocapillary tangent stress, $\mu$ - is the dynamics viscosity, $\rho$ - is the density, $\mathbf{g}$ - is the gravity vector, $\mathrm{h}$ - is the film thickness, $\sigma$ - is the surface tension, $\sigma \mathrm{T}-$ is the linear coefficient, which determines the dependence of the surface tension on 
temperature, $T$ - is the temperature, $H=\frac{h_{r r}}{\left(1+h_{r}^{2}\right)^{3 / 2}}+\frac{h_{r}}{r\left(1+h_{r}^{2}\right)^{1 / 2}}-$ is the double mean curvature.

The temperature distribution in the liquid layer is determined by the energy equation in cylindrical coordinates:

$$
\rho C_{p}\left(\frac{\partial T}{\partial t}+u \frac{1}{r} \frac{\partial\left(r T_{r}\right)}{\partial r}+v \frac{\partial T_{z}}{\partial z}\right)=\frac{1}{r} \frac{\partial}{\partial r}\left(\lambda\left(r \frac{\partial T_{r}}{\partial r}\right)\right)+\frac{\partial^{2} T_{z}}{\partial z^{2}}+Q
$$

where, $Q=Q(t, r, z)=\left\{\begin{array}{l}Q=0 \text {, outside the heater } \\ Q=\text { const }>0 \text {, in the heater }\end{array}\right.$ - is the bulk density of the heat sources, $\lambda$ - thermal conductivity coefficient; $C p$ - is the specific heat; $\rho$ - is the density of the liquid.

System of equations (2)-(3) is the direct problem of thermocapillary convection. Equation (3) is a nonlinear differential equation of the first order with respect to time, and a fourth order in spatial variables with respect to the unknown function $h(t, r)$. Numerical simulation of inverse problem is based on results obtained from solving the direct problem of thermocapillary convection $[4,5,6]$.

\section{Solving algorithm}

To find the numerical solution of the direct problem (2) - (3) we used the algorithm for joint solving the energy equation (3) and the evolution equation (2). Stationary solutions are obtained by the establishment method $[5,6]$.

The algorithm for solving inverse problem is based on solving problem of minimizing the residual function, which expresses the integral deviation of the measured response, and subsequent calculations. The minimum is found numerically by the Newton's method.

Residual function is given as:

$$
J\left(\sigma^{k}\right)=\frac{1}{t_{1}} \int_{0}^{t_{1}}\left[d S p\left(\sigma^{*}\right)-d \operatorname{Sp}\left(\sigma^{k}, t\right)\right]^{2} d t \geq 0
$$

where $d S p$ - is the function of thermocapillary response, $\sigma^{*}$ - is the exact value of surface tension coefficient, measurement time $\mathrm{t}_{1}=5 \mathrm{~s}$.

The problem of function minimization is formulated as:

$$
J\left(\sigma^{k}\right) \rightarrow \min _{\sigma^{k} \in\left[\sigma_{\min }, \sigma_{\max }\right]}
$$

\section{Numerical results}

Numerical calculations have been made for silicone oil (dimethylpolysiloxane) type PMS-5. Silicone oil PMS-5 is a non-volatile liquid, so evaporation was not taken into account. Figures 1(b, c) show calculated thermocapillary responses for different values of the coefficients $\sigma$ and $\sigma \mathrm{T}$. It can be seen that for different $\sigma$ or $\sigma \mathrm{T}$ thermocapillary responses (the diameters of the reflected laser beam) are significantly different. It means that the system is quite sensitive to the liquid properties $\sigma$ and $\sigma \mathrm{T}$.

The results of the calculated functions $J(\sigma)$ and $J\left(\sigma_{T}\right)$ for layer PMS-5 are shown in Fig.2. It can be seen that the functions are "good" in terms of solving the inverse problem: the shapes of functions are convex and have unique minimum. This allows finding the minimum functions by Newton method for several iterations with a sufficiently high accuracy. 

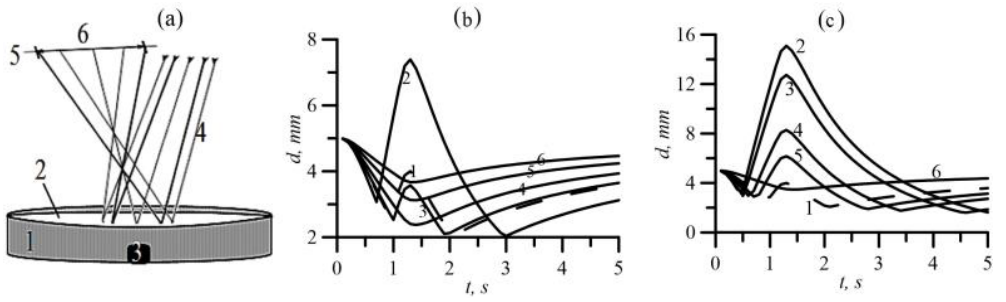

Fig. 1. (a) System scheme: 1 - Teflon cuvette, $d=32 \mathrm{~mm}, 2$ - PMS-5, 3 - heater, $r=1.6 \mathrm{~mm}$, $\mathrm{Q}=0.00165 \mathrm{~W}, 4$ - laser beam, 5 - screen, 6 - diameter of the light spot. (b) Thermocapillary response depending on different $\sigma$ for PMS-5, $\mathrm{h}_{0}=238 \mu \mathrm{m}: 1-$ exact solution $\sigma=\sigma^{*}=0.01827 \mathrm{~N} / \mathrm{m}, 2$ $-\sigma=0.01 \mathrm{~N} / \mathrm{m}, 3-\sigma=0.02 \mathrm{~N} / \mathrm{m}, 4-\sigma=0.03 \mathrm{~N} / \mathrm{m}, 5-\sigma=0.05 \mathrm{~N} / \mathrm{m}, 6-\sigma=0.08 \mathrm{~N} / \mathrm{m}$. (c)

Thermocapillary response depending on different $\sigma_{\mathrm{T}}$ with the same conditions: $1-$ exact solution $\sigma=$ $\sigma_{\mathrm{T}}{ }^{*}=-0.066 \times 10^{-3} \mathrm{~N} /(\mathrm{mxK}), 2-\sigma_{\mathrm{T}}=-0.16 \times 10^{-3} \mathrm{~N} /(\mathrm{mxK}), 3-\sigma_{\mathrm{T}}=-0.141 \times 10^{-3} \mathrm{~N} /(\mathrm{mxK}), 4-\sigma_{\mathrm{T}}=-$ $0.104 \times 10^{-3} \mathrm{~N} /(\mathrm{mxK}), 5-\sigma \mathrm{T}=-0.08 \times 10-3 \mathrm{H} /(\mathrm{mxK}), 6-\sigma_{\mathrm{T}}=-0.03 \times 10^{-3} \mathrm{~N} /(\mathrm{mxK})$.
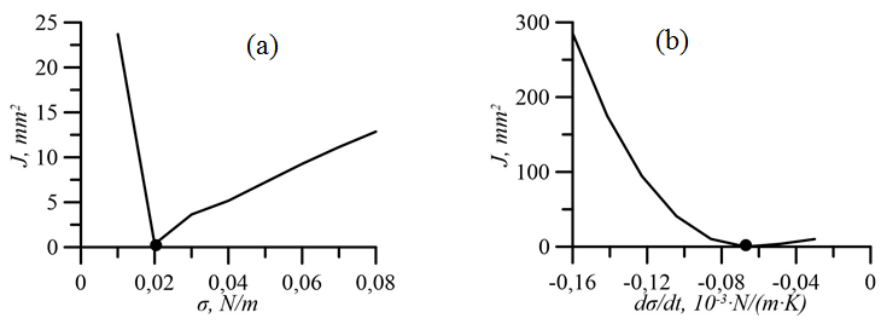

Fig. 2. Residual function for PMS-5, $\mathrm{h}_{0}=238 \mu \mathrm{m}$, (a) $J(\sigma)$, •- exact $\sigma^{*}$, (b) $J\left(\sigma_{T}\right)$, •- exact $\sigma_{\mathrm{T}}{ }^{*}$

\section{Conclusion}

We have developed algorithms and created calculating code for solving inverse problem to identify properties of liquid: $\sigma$ and $\sigma_{T}$. Thermocapillary response function is used as additional information for solving inverse problem. Calculation results show that the thermocapillary response is quite sensitive to the liquid properties $\sigma$ and $\sigma_{\mathrm{T}}$. It means that using of the thermocapillary response as additional data of this inverse problem is justified and reliable. The developed algorithms have been tested on synthetic data, which has been obtained from the solution of direct problems. The developed algorithm has shown a good convergence and efficiency.

The study was financially supported by the Russian Science Foundation (Project No. 15-19-20049).

\section{References}

1. I.V. Marchuk, O.A Kabov, Doklady Physics 61, 1 (2016)

2. B.A. Bezuglyi, A.A. Fedorets, O.A. Tarasov, N.A. Ivanova, Surface, X-Ray, Synchrtronic and Neytronic Studies 6, 80 (2004) [in Russian]

3. B.A. Bezuglyi, A.A. Fedorets, Technical Physics Letters 27, 359 (2001)

4. E.V. Barakhovskaia, I.V. Marchuk, A.A Fedorets, J. Physics: Conference Series, 754 (2016)

5. I.V. Marchuk, J. Eng. Thermophys. 24, 381 (2015)

6. A. Oron, S.H. Davis, S.G. Bankoff, Rev. Modern Physics 69, 3 (1997) 